\title{
Ideación suicida y estrategias de afrontamiento frente al embarazo precoz en un hospital de Lima Metropolitana
}

\author{
Suicidal ideation and coping strategies against early pregnancy in a hospital in \\ metropolitan Lima
}

\author{
Gerson Hilaquita Cruz¹, Yomira Palacios Ponce de León², Rocío Evelin Herrera Blancas³
}

\begin{abstract}
RESUMEN
Objetivo: Determinar la relación entre ideación suicida y estrategias de afrontamiento frente al impacto del embarazo en adolescentes de un hospital nacional de Lima Metropolitana. Material y Métodos: Se utilizó un diseño no experimental, de corte transversal y alcance correlacional. Se llevó a cabo en un grupo de 140 adolescentes gestantes entre 13 y 18 años. Los instrumentos usados fueron la Escala de Ideación Suicida (SSI) de Beck, adaptada en el Perú por Eugenio y Zelada (2011) y la Escala de Afrontamiento para Adolescentes (ACS) de Frydenberg y Lewis (2000). Resultados: Los resultados obtenidos demuestran que existe una relación negativa altamente significativa entre ideación suicida y quince de las estrategias de afrontamiento $\left(r h o=-0.562^{* *} p<0.05\right)$. Conclusiones: Por lo tanto, se concluye que a mayor uso de las estrategias de afrontamiento, menor riesgo de ideación suicida y viceversa.
\end{abstract}

Palabras clave: Ideación suicida, estrategias de afrontamiento, gestantes, adolescentes.

\begin{abstract}
Objective: The objective of this research is to determine the relationship between suicidal ideation and coping strategies against pregnancy in adolescent mothers in a national hospital in metropolitan Lima. Material and Methods: A non - experimental, cross - sectional correlational design was used. It was carried out in a group of 140 pregnant adolescents between 13 and 18 years old. The instruments used were the Suicidal Ideation Scale (SSI) of Beck, adapted in Peru by Eugenio and Zelada (2011) and the Adolescent Coping Scale (ACS) of Frydenberg and Lewis, adapted by Canessa in 2002. Results: The results obtained show that there is a highly significant negative relationship between suicidal ideation and fifteen of the coping strategies ( $r h o=-0.562{ }^{* *} p<0.05$ ). Conclusions: Therefore, it is concluded that the greater the use of coping strategies, the lower the risk of suicidal ideation and vice versa.
\end{abstract}

Keywords: Suicidal ideation, Coping strategies, Pregnancy, Pregnant women, Adolescents

\footnotetext{
${ }^{1}$ Evaluador de Proyecto. Instituto Nacional de Salud Mental, Hospital Honorio Delgado Hideyo Noguchi, Lima, Perú. ${ }^{2}$ Psicóloga, Hospital Nacional Docente Madre Niño San Bartolomé, Lima, Perú.

${ }^{3}$ Psicóloga del Centro de Salud Aurahua I-III, Huancavelica.
} 


\section{INTRODUCCIÓN}

En los últimos años, el incremento del índice de maternidad adolescente, ya sea como consecuencia de una violación sexual o una relación "amorosa" precipitada, ha sido y sigue siendo un motivo de real preocupación, dado que este hecho suele interferir en el desarrollo de una etapa, donde todavía no se alcanza la madurez física ni mental. En el Perú, según la Encuesta Demográfica y de Salud Familiar (ENDES 2014), elaborada por el INEI, el embarazo precoz se ha incrementado de $12.5 \%$ (2011) a $14.6 \%$ (2014) principalmente en zonas de la selva y el norte del país, lo que afecta significativamente la tasa de mortalidad materna. Esta situación empeora cuando ellas viven en un medio familiar generalmente poco receptivo para aceptar y proteger a la gestante (Della, 2006).

Las estadísticas del Seguro Integral de Salud (SIS) mencionan que, desde el año 2012, más de 150 mil niñas y adolescentes, entre 9 y 17 años, resultaron embarazadas, esto significa que el SIS atendió diariamente de 68 a 70 niñas, púberes y adolescentes, dicho porcentaje pertenece únicamente a casos vistos por esta entidad, forman este porcentaje solo casos reportados. Ciertamente estas cifras son alarmantes y a la vez recientes, puesto que en el 2017 se reportaron 304 casos de niñas embarazadas (La República, 2018). Por otro lado, El Fondo de Población de las Naciones Unidas - UNFPA (2013) menciona que los problemas de salud son más probables si la niña queda embarazada en los dos años posteriores a la menarquía o cuando su pelvis y canal de parto todavía está en crecimiento, en consecuencia, aumenta la probabilidad de mortandad. Las madres menores de 16 años tienen cuatro veces más riesgos de morir que una mujer de 20 años.

Por otro lado, la Organización Mundial de la Salud (OMS, 2017) señala que cada día fallecen más de 3000 adolescentes (es decir, un millón 200000 muertes anuales) y que una de las causas es el suicidio. Al respecto, El Fondo de Población de las Naciones Unidas (UNICEF), señaló que cada año aproximadamente 71000 adolescentes se quitan la vida, y una cifra 40 veces superior, intentó hacerlo. Algunos factores desencadenantes del suicidio, en algunas de estas adolescentes, son la depresión asociada a un embarazo no deseado, la exclusión social por la edad de la gestante, maltratos en la infancia, violencia doméstica, entre otros. Cabe señalar, además, que la mayor parte de estos factores podría evitarse (citado por Lepineux, 2016).

Esto evidencia la carencia de recursos en las adolescentes para poder afrontar situaciones desesperantes, debido a lo cual piensan que la única salida es la muerte o un aborto. Como el caso de Estela (el nombre ha sido cambiado) de 14 años, quien fue víctima de abuso sexual y resultó embarazada, decidió tener su primer hijo y trató de superar esa situación. Tiempo después quedó embarazada por segunda vez (por violación); frente a esta situación estresante decidió dejar los estudios y se alejó de sus compañeros, de sus amigos y de su familia; finalmente se suicidó. Otro caso similar es el de Ángela, menor quien resultó embarazada cuando tenía 13 años, frente a esta situación decidió abortar. Ella llegó al hospital en estado crítico y falleció tres días después por una infección generalizada que se complicó con insuficiencia renal (Perú 21, 2016). Esta realidad aún no se ve en su totalidad, puesto que no todos los casos son reportados oficialmente.

Algunos autores mencionan que acercarse a comprender este fenómeno es sumamente complejo, dado que el hecho de quitarse la vida es la culminación de un plan que parte del pensamiento suicida; de no detectarse a tiempo puede acabar con el futuro de quien lo comete (Arcos, 2016). Por lo que la ideación suicida es definida como todo pensamiento voluntario autodestructivo, que comprende ideas negativas hacia la vida; estos pensamientos pueden o no contener una planificación (cómo, cuándo y dónde llevar a cabo la conducta de autodestrucción) del acto suicida (Robledo, 2007). Asimismo, Eugenio y Zelada (2011) consideran la ideación suicida como todo pensamiento persistente, planificado de querer cometer suicidio.

Existen diversos factores asociados a la ideación suicida como la depresión, baja autoestima, ansiedad, trastornos mentales, abuso sexual, embarazo no planificado, violencia familiar y la falta de estrategias de afrontamiento, entre otros (Clemente y Gonzáles 1996; Freydenberg y Lewis 2000; Vera y Díaz 2012; Zevallos 2015).

Los estudios anteriores señalan la relación entre la forma cómo se enfrentan los problemas y el desarrollo de ideas suicidas. La combinación 
de estas dos variables cobra importancia debido a que puede influir en la aparición de conductas de mayor riesgo, como es el intento suicida, particularmente en una etapa como la adolescencia, siendo las mujeres las que presentan mayor vulnerabilidad a este fenómeno (Serrano \& Flores, 2005). Al respecto, Frydenberg y Lewis (2000) afirman que las estrategias de afrontamiento son un conjunto de acciones cognitivas y afectivas que realiza el individuo, en respuesta a una preocupación en particular con la finalidad de restaurar su propio equilibrio, Por esta razón, la adolescente en su intento de restaurar el equilibrio o reducir la turbulencia, deberá resolver el problema, cambiando el estímulo o acomodándose a la preocupación, sin necesariamente dar una solución. (Citado por Canessa, 2002).

Cassaretto, Chau, Oblitas y Valdez (2003) señalan que la función principal del afrontamiento es regular aspectos que alteren el área emocional, para así conseguir resultados positivos en el bienestar y evitando el incremento de riesgos de mortandad y morbilidad, por lo tanto, el afrontamiento interactúa con la situación y capacidad que posee la persona para sobrellevar las diversas situaciones adversas tratando de no evitarlas o escapar.

La maternidad trae consigo diferentes cambios cognitivos, conductuales y emocionales que necesitan ser aceptados por la joven madre quien probablemente no esté preparada, sin embargo, deberá utilizar recursos que ayuden en la adaptación de estos cambios. Ahora bien, no únicamente son los cambios mencionados que perturban a la gestante adolescente, sino también elementos como carencia económica, ausencia del apoyo de pareja, rechazo familiar y social, frustración y baja tolerancia.

Frente a ello, el embarazo precoz es considerado como una situación estresante, debido a la fragilidad e inestabilidad propia de esta etapa y a la demanda familiar, social a las que se ve expuesta la adolescente (Sorto, López y Velázquez, 2013). He ahí la necesidad de estudiar, en las adolescentes gestantes, la utilización de recursos y estrategias que son usados para afrontar su situación; este estudio permitirá evidenciar la forma de actuar que tienen frente a su realidad.

Frente a este panorama se presenta este estudio, con el objetivo de determinar la relación entre ideación suicida y estrategias de afrontamiento, frente al impacto del embarazo en adolescentes.

Este estudio es relevante, ya que permite ahondar conocimientos y obtener datos contundentes que describen la relación entre ideación suicida y estrategias de afrontamiento, frente al embarazo en gestantes adolescentes. Esto con el propósito de conocer si las adolescentes presentan ideación suicida frente al embarazo inesperado, como también se podrá medir los diferentes recursos de afronte que cada adolescente utiliza ante la situación que vive. Asimismo, se identificó la relación entre las variables, consolidando nuevos estudios e investigaciones en nuestra ciudad, más aún en la población descrita. Por otro lado, este estudio se justifica, a nivel teórico, porque brinda información actualizada sobre el tema propuesto: ideación suicida y estrategias de afrontamiento en gestantes adolescentes, puesto que hay carencias de estudio de correlación de estas dos variables en el Perú.

En cuanto al aporte social, esta investigación pretende internalizar y procesar las experiencias desagradables que viven las adolescentes gestantes y contribuir a los lineamientos de la política de salud, buscando disminuir la prevalencia de ideación suicida frente al embarazo en las adolescentes, mediante educación, tanto para los padres como para las adolescentes.

Los resultados de este estudio ampliarán otros puntos de investigación, a su vez permitirán planificar programas de intervención y estudios explicativos, con la finalidad de mejorar las estrategias de afrontamiento y disminuir la prevalencia de ideación suicida.

En los últimos años, el incremento del índice de maternidad adolescente, ya sea como consecuencia de una violación sexual o una relación "amorosa" precipitada, ha sido y sigue siendo un motivo de real preocupación, dado que este hecho suele interferir en el desarrollo de una etapa, donde todavía no se alcanza la madurez física ni mental. En el Perú, según la Encuesta Demográfica y de Salud Familiar (ENDES 2014), elaborada por el INEI, el embarazo precoz se ha incrementado de $12.5 \%$ (2011) a $14.6 \%$ (2014) principalmente en zonas de la selva y el norte del país, lo que afecta significativamente la tasa de mortalidad materna. Esta situación empeora cuando ellas 
viven en un medio familiar generalmente poco receptivo para aceptar y proteger a la gestante (Della, 2006).

Las estadísticas, del Seguro Integral de Salud (SIS), mencionan que desde el año 2012 más de 150 mil niñas y adolescentes entre 9 y 17 años resultaron embarazadas, esto significa que el SIS atendió diariamente de 68 a 70 niñas púberes y adolescentes, dicho porcentaje pertenece únicamente a casos vistos por esta entidad, forman este porcentaje solo casos reportados. Ciertamente estas cifras son alarmantes y a la vez recientes, puesto que en el 2017 se reportaron 304 casos de niñas embarazadas (La República, 2018). Por otro lado, El Fondo de Población de las Naciones Unidas - UNFPA (2013) menciona que los problemas de salud son más probables si la niña queda embarazada en los dos años posteriores a la menarquía o cuando su pelvis y canal de parto todavía está en crecimiento, en consecuencia, aumenta la probabilidad de mortandad. Las madres menores de 16 años tienen cuatro veces más riesgos de morir que una mujer de 20 años.

Por otro lado, la Organización Mundial de la Salud (OMS, 2017) señala que cada día fallecen más de 3000 adolescentes (es decir, un millón 200000 muertes anuales) y que una de las causas es el suicidio. Al respecto, El Fondo de Población de las Naciones Unidas

(UNICEF), señaló que cada año aproximadamente 71000 adolescentes se quitan la vida, y una cifra 40 veces superior, intentó hacerlo. Algunos factores desencadenantes del suicidio en algunos de estos adolescentes son la depresión asociada a un embarazo no deseado, la exclusión social por la edad de la gestante, maltratos en la infancia, violencia doméstica, entre otros. Cabe señalar, además, que la mayor parte de estos factores podría evitarse (citado por Lepineux, 2016).

Esto evidencia la carencia de recursos en las adolescentes para poder afrontar situaciones desesperantes, debido a lo cual piensan que la única salida es la muerte o un aborto. Como el caso de Estela (el nombre ha sido cambiado) de 14 años, quien fue víctima de abuso sexual y resultó embarazada, decidió tener su primer hijo y trató de superar esa situación. Tiempo después quedó embarazada por segunda vez (por violación); frente a esta situación estresante decidió dejar los estudios y se alejó de sus compañeros, de sus amigos y de su familia; finalmente se suicidó. Otro caso similar, es el de Ángela, menor que resultó embarazada cuando tenía 13 años, frente a esta situación decidió abortar. Ella llegó al hospital en estado crítico y falleció tres días después por una infección generalizada que se complicó con insuficiencia renal (Perú 21, 2016). Esta realidad aún no se ve en su totalidad puesto que no todos los casos son reportados oficialmente.

\section{MATERIALES Y MÉTODOS}

El diseño de la investigación es no experimental porque no se manipularon las variables, de corte trasversal ya que los datos fueron tomados en un momento dado, con el objetivo de revisar su interrelación en ese momento. Asimismo, es de alcance correlacional habiendo sido su principal interés describir niveles de ideación suicida y estrategias de afrontamiento y analizar la relación entre las variables mencionadas (Hernández, Fernández y Baptista, 2010).

\section{Participantes}

Por lo expuesto, la muestra escogida provino de una selección a conveniencia de 140 adolescentes gestantes de un hospital de Lima Metropolitana, cuyas edades fueron entre 13 y 18 años. 
Tabla 1

Datos sociodemográficos

\begin{tabular}{|c|c|c|}
\hline Variables & $\mathbf{n}$ & $\%$ \\
\hline $\begin{array}{l}\text { Edad: } \\
\text { Adolescencia Temprana: } 10 \text { a } 14 \text { años. } \\
\text { Adolescencia Tardía: } 15 \text { a } 19 \text { años }\end{array}$ & $\begin{array}{l}19 \\
121\end{array}$ & $\begin{array}{l}14 \\
86\end{array}$ \\
\hline $\begin{array}{l}\text { Edad de embarazo: } \\
\text { Adolescencia Temprana: } 10 \text { a } 14 \text { años. } \\
\text { Adolescencia Tardía: } 15 \text { a } 19 \text { años }\end{array}$ & $\begin{array}{l}33 \\
107\end{array}$ & $\begin{array}{l}23.6 \\
76.4\end{array}$ \\
\hline $\begin{array}{l}\text { Estado civil: } \\
\text { Soltera } \\
\text { Conviviente } \\
\text { Casada }\end{array}$ & $\begin{array}{l}102 \\
33 \\
5\end{array}$ & $\begin{array}{l}72.9 \\
23.6 \\
3.6\end{array}$ \\
\hline $\begin{array}{l}\text { Apoyo pareja: } \\
\text { Sí } \\
\text { No }\end{array}$ & $\begin{array}{l}57 \\
83\end{array}$ & $\begin{array}{l}40.7 \\
59.3\end{array}$ \\
\hline $\begin{array}{l}\text { Apoyo familia } \\
\text { Sí } \\
\text { No }\end{array}$ & $\begin{array}{l}70 \\
70\end{array}$ & $\begin{array}{l}50 \\
50\end{array}$ \\
\hline $\begin{array}{l}\text { Grado de instrucción: } \\
\text { Primaria } \\
\text { Secundaria } \\
\text { Secundaria incompleta } \\
\text { Superior }\end{array}$ & $\begin{array}{l}2 \\
63 \\
73 \\
3\end{array}$ & $\begin{array}{l}1.4 \\
44.3 \\
52.1 \\
2.1\end{array}$ \\
\hline $\begin{array}{l}\text { Procedencia : } \\
\text { Costa } \\
\text { Sierra } \\
\text { selva }\end{array}$ & $\begin{array}{l}54 \\
44 \\
42\end{array}$ & $\begin{array}{l}38.6 \\
31.4 \\
30\end{array}$ \\
\hline $\begin{array}{l}\text { Relación de padres: } \\
\text { Armoniosa } \\
\text { Conflictiva } \\
\text { Distante }\end{array}$ & $\begin{array}{l}13 \\
82 \\
45\end{array}$ & $\begin{array}{l}9.3 \\
58.6 \\
32.1\end{array}$ \\
\hline
\end{tabular}

En la tabla 1 se observa que el $86 \%$ de la población pertenece al grupo de edad entre 10 a 14 años, mientras que el $14 \%$ pertenece al grupo de 15 a 19 años. Asimismo de esta población el $76.4 \%$ inició su gestación en la adolescencia tardía (15-19 años) y el $23.6 \%$ en la adolescencia temprana (10-14 años). En relación al apoyo que recibieron, el $40.7 \%$ refiere que recibió apoyo de su pareja y el 50\% recibió apoyo de su familia. Respecto a la percepción sobre la relación de sus padres, el $58.6 \%$ de las adolescentes refieren que sus padres mantienen una relación conflictiva, el $32.1 \%$ una relación distante y solo el $9.3 \%$ una relación armoniosa.

\section{Instrumentos}

Los instrumentos utilizados fueron la Escala de Ideación Suicida (SSI), elaborada por Beck en 1979, para cuantificar y evaluar la intencionalidad suicida o el grado de seriedad e intensidad con el que alguien pensó o está pensando suicidarse; para esta investigación se empleó la adaptación de dicha escala realizada por Eugenio y Zelada en el 2011. La consistencia interna de la escala fue de 0.82 , índice que coincide con Eugenio y Zelada (a 0.79). De igual manera, se encuentra consistencia interna por dimensiones. Por lo tanto, el instrumento es confiable. La Escala de Ideación Suicida puede administrarse de manera individual o colectiva y consta de 18 ítems, de los cuales su puntuación oscila entre 0,1 y 2 . 
Posee 4 dimensiones: Actitud hacia la vida/ muerte, pensamientos/deseos suicidas, proyecto de intento suicida, desesperanza. Está formada por una parte objetiva: ítems del 1 al 8 que explora las circunstancias objetivas relacionadas con la tentativa de suicidio. Una parte subjetiva: ítems del 9 al 15, la cual valora las expectativas durante la tentativa. $Y$ otros aspectos: ítems del 16 al 18, que evalúa la situación de llevar a cabo un intento de suicidio. Posteriormente se procedió a la codificación de cada una de las respuestas, primero se sacó los puntajes por dimensiones, para luego obtener la puntuación total de la escala general, la cual es la suma de las puntuaciones de los ítems 1 al 18; esto se interpreta de la siguiente manera: de 0 a 17 lo que equivale a un nivel bajo, de 18 a 30 a un nivel medio, y de 31 a 36 a un nivel alto.

Para medir las estrategias de afrontamiento se empleó la Escala de Afrontamiento para adolescentes de Frydemberg y Lewis, adaptada por Beatriz Canessa en el 2002, con validez ( $p$ $<0.01$ ) y confiabilidad del constructo $(0.79)$. El instrumento está compuesto por 80 elementos o ítems, que permiten evaluar con fiabilidad de 18 diferentes estrategias de afrontamiento que se han identificado conceptual y empíricamente. Asimismo, dicha escala se aplica a adolescentes entre 12 y 18 años de edad, pudiendo ser administrado de forma individual o colectiva con un tiempo aproximado de 15 minutos. Las 18 estrategias están agrupadas en tres dimensiones o estilos básicos de afrontamiento: La primera llamada "resolución del problema", orientada a identificar con cuánta frecuencia el evaluado aborda la situación estresante, con la finalidad de buscar una solución. La segunda orientada a la "búsqueda de ayuda en los demás", esta dimensión investiga si es frecuente entre los evaluados compartir sus preocupaciones y buscar soporte en terceras personas como amigos y familiares. Y la tercera dimensión, denominada "afrontamiento improductivo", donde el individuo no encuentra una solución a pesar de las estrategias usadas. Los 80 elementos cerrados se puntúan mediante una escala de tipo Likert de cinco puntos: (nunca lo hago, lo hago raras veces, lo hago algunas veces, lo hago a menudo y lo hago con mucha frecuencia). La puntuación se obtiene mediante la suma total de los puntos obtenidos en cada uno de los 5, 4 ó 3 elementos que consigna una escala o estrategia de afrontamiento, luego el puntaje se multiplica por 4, 5 ó 7 ítems, de acuerdo al número de elementos de cada escala.

\section{Análisis de datos}

Para el análisis de datos se utilizó el software estadístico SPSS (Statistical Package for the Social Sciences versión 24.0), una vez recolectada la información se la transfirió a la matriz de datos del software estadístico para su respectivo análisis, los resultados que se obtuvieron fueron ordenados en tablas, cuya interpretación permitió evaluar y verificar la hipótesis planteada, para ello se utilizó el proceso estadístico Sperman que permitió determinar si existe relación significativa entre ambas variables.

\section{RESULTADOS}

En la tabla 2 se observa que el $4.3 \%$ de las adolescentes, de manera general, presentaron niveles altos de ideación suicida, es decir, en el momento que se enteraron de su estado de gestación, pensaron y hasta planificaron una acción suicida específica, creyendo no encontrar otra solución y sintiéndose desesperanzadas. Mientras que el $36.4 \%$ evidenció estos síntomas o pensamiento suicidas en niveles moderados. Y por último, el 59.3\% presentó niveles bajos de ideación suicida. Respecto a las dimensiones, los resultados obtenidos demuestran que el $65.7 \%$ presentó niveles moderados en la dimensión actitud hacia la muerte; lo cual indica que demostraron ambivalencia en relación a la vida/muerte. El $20.7 \%$ presentó niveles altos de pensamiento suicida; es decir, pensamientos negativos continuos y autocontrol deteriorado. Por otro lado, el $28.6 \%$ ha considerado el intento suicida, sin detalles específicos e inseguridad para concretar el acto. Finalmente, el $20.7 \%$ presentó desesperanza realizando preparativos finales, sin embargo, no llegaron a concretar el suicidio. 
Tabla 2

Niveles de ideación suicida y sus dimensiones

\begin{tabular}{lllllll} 
& \multicolumn{3}{c}{ Bajo } & \multicolumn{3}{c}{ Medio } \\
\cline { 2 - 7 } & $\mathrm{n}$ & $\%$ & $\mathrm{n}$ & $\%$ & $\mathrm{n}$ & $\%$ \\
\hline Ideación suicida & 83 & 59.3 & 51 & 36.4 & 6 & 4.3 \\
Actitud hacia la muerte & 24 & 17.1 & 92 & 65.7 & 24 & 17.1 \\
Pensamiento suicida & 55 & 39.3 & 56 & 40 & 29 & 20.7 \\
Proyecto suicida & 87 & 62.1 & 40 & 28.6 & 13 & 9.3 \\
Desesperanza & 107 & 76.4 & 29 & 20.7 & 4 & 2.9 \\
\hline
\end{tabular}

En la tabla 3 se aprecia las estrategias que emplearon las adolescentes frente al impacto del embarazo. En cuanto a las estrategias de "resolución del problemas" se observa que, en su mayoría, las gestantes raras veces buscaron concentrarse en resolver el problema $(35.7 \%)$, esforzarse y tener éxito $(41.4 \%)$ y buscar diversiones relajantes (55.7\%), es decir, hallar una solución productiva frente a la situación estresante. Sin embargo, se observó que, a menudo, el $21.4 \%$ utilizó las estrategias de "fijarse en lo positivo y distracción física", es decir, trataron de buscar el lado positivo de la situación, mientras que otras realizaron deportes o actividades físicas, tratando de sobrellevar la situación. Respecto a las estrategias utilizadas, en relación con los demás, el $37.1 \%$ de las participantes refirieron que, a menudo, buscaron "apoyo espiritual", empleando la oración o rezo como medio de fortaleza, mientras que el $44.3 \%$ confirmó que raras veces buscó "apoyo social”, rechazando hablar con otros, buscar ánimo y sostén socioemocional en ese momento. Referente a las estrategias improductivas se observa que, en su mayoría, las adolescentes recurrieron al uso de la evitación, donde el $35.7 \%$ de gestantes utilizaron la "reducción de la tensión", es decir, buscaron aliviar la situación bebiendo alcohol o utilizando otras drogas y el $45 \%$ prefirió "reservar sus problemas para sí", aislándose de los demás y no deseando que conozcan su problema. El $77.1 \%$ utilizó algunas veces la estrategia "falta de afrontamiento" la cual evidencia la incapacidad para enfrentar la situación estresante; el $74.3 \%$ decidió "hacerse ilusiones", esperando que se reviertan los resultados y ocurra algo mejor; el $70.7 \%$ recurrió a "preocuparse", sintiendo temor hacia el futuro. Por último, el 49.3\% mencionó que raras veces "intentaron ignorar el problema" (negar el embarazo).

Tabla 3

Niveles de estrategias de afrontamiento

\begin{tabular}{|c|c|c|c|c|c|c|c|c|c|c|}
\hline \multirow[t]{2}{*}{ No utiliza } & \multicolumn{4}{|c|}{$\begin{array}{l}\text { Raras } \\
\text { veces }\end{array}$} & \multicolumn{2}{|c|}{$\begin{array}{l}\text { Algunas } \\
\text { veces }\end{array}$} & \multicolumn{2}{|c|}{$\begin{array}{l}\text { A } \\
\text { menudo }\end{array}$} & \multicolumn{2}{|c|}{$\begin{array}{l}\text { Con mucha } \\
\text { frecuencia }\end{array}$} \\
\hline & $n$ & $\%$ & $n$ & $\%$ & $n$ & $\%$ & $n$ & $\%$ & $n$ & $\%$ \\
\hline \multicolumn{11}{|l|}{ Resolución del problema } \\
\hline $\begin{array}{l}\text { Concentrarse en resolver el } \\
\text { problema }\end{array}$ & 3 & 2.1 & 50 & 35.7 & 77 & 55.0 & 10 & 7.1 & 0 & 0 \\
\hline Esforzarse y tener éxito & 2 & 1.4 & 58 & 41.4 & 69 & 49.3 & 11 & 7.9 & 0 & 0 \\
\hline Invertir en amigos íntimos & 0 & 0 & 20 & 14.3 & 89 & 63.6 & 28 & 20 & 3 & 2.1 \\
\hline Buscar pertenencia & 1 & 0.7 & 52 & 37.1 & 76 & 54.3 & 11 & 7.9 & 0 & 0 \\
\hline Fijarse en lo positivo & 1 & 0.7 & 37 & 26.4 & 70 & 50 & 30 & 21.4 & 2 & 1.4 \\
\hline Buscar diversiones relajantes & 18 & 12.9 & 78 & 55.7 & 30 & 21.4 & 14 & 10 & 0 & 0 \\
\hline Distracción física & 0 & 0 & 39 & 27.9 & 71 & 50.7 & 30 & 21.4 & 0 & 0 \\
\hline \multicolumn{11}{|l|}{ Relación con los demás } \\
\hline Buscar apoyo social & 0 & 0 & 62 & 44.3 & 72 & 51.4 & 6 & 4.3 & 0 & 0 \\
\hline Acción social & 0 & 0 & 25 & 17.9 & 99 & 70.7 & 16 & 11.4 & 0 & 0 \\
\hline
\end{tabular}




\begin{tabular}{|c|c|c|c|c|c|c|c|c|c|c|}
\hline Buscar apoyo espiritual & 0 & 0 & 13 & 9.3 & 74 & 52.9 & 52 & 37.1 & 1 & 0.7 \\
\hline Buscar ayuda profesional & 2 & 1.4 & 46 & 32.9 & 79 & 56.4 & 12 & 8.6 & 1 & 0.7 \\
\hline \multicolumn{11}{|l|}{ Afrontamiento improductivo } \\
\hline Preocuparse & 0 & 0 & 25 & 17.9 & 99 & 70.7 & 14 & 10 & 2 & 1.4 \\
\hline Hacerse ilusiones & 0 & 0 & 25 & 17.9 & 104 & 74.3 & 11 & 7.9 & 0 & 0 \\
\hline Falta de afrontamiento & 0 & 0 & 8 & 5.7 & 108 & 77.1 & 24 & 17.1 & 0 & 0 \\
\hline Ignorar el problema & 2 & 1.4 & 69 & 49.3 & 69 & 49.3 & 0 & 0 & 0 & 0 \\
\hline Reducción de la tensión & 0 & 0 & 7 & 5.0 & 80 & 57.1 & 50 & 35.7 & 3 & 2.1 \\
\hline Reservarlo para sí & 0 & 0 & 10 & 7.1 & 56 & 40 & 63 & 45.0 & 11 & 7.9 \\
\hline Autoinculparse & 0 & 0 & 38 & 27.1 & 91 & 65.0 & 11 & 7.9 & 0 & 0 \\
\hline
\end{tabular}

\section{Prueba de normalidad}

Con el propósito de realizar los análisis comparativos y contrastar las hipótesis planteadas, se procedió a realizar, primero, la prueba de bondad de ajuste para precisar si las variables presentan una distribución normal. En este sentido, la tabla 4 presenta los resultados de la prueba de bondad de ajuste de Kolmogorov - Smirnov (K-S). Los datos correspondientes a las variables, en su totalidad y en su mayoría, no presentan una distribución normal dado que el coeficiente obtenido (K-S) es significativo $(p<0.05)$. Por tanto, para los análisis estadísticos correspondientes se empleará estadística no paramétrica.

Tabla 4

Prueba de bondad de ajuste a la curva normal para las variables de estudio

\begin{tabular}{|c|c|c|c|c|c|}
\hline Instrumentos & Variables & Media & D.E. & K-S & $\mathbf{p}$ \\
\hline \multirow[t]{8}{*}{ Ideación suicida } & Ideación Suicida Global & 16.41 & 7.290 & 0.073 &, $068 c$ \\
\hline & Buscar apoyo social & 51.7714 & 8.56658 & 0.204 &, $000 c$ \\
\hline & $\begin{array}{l}\text { Concentrarse en resolver el } \\
\text { problema }\end{array}$ & 52.7143 & 10.46012 & 0.149 &, $000 c$ \\
\hline & Esforzarse y tener éxito & 53.0286 & 10.93469 & 0.106 &, $001 c$ \\
\hline & Preocuparse & 59.06 & 10.906 & 0.132 &, $000 c$ \\
\hline & Invertir en amigos íntimos & 62.37 & 11.128 & 0.099 & ,002c \\
\hline & Buscar pertenencia & 54.86 & 11.448 & 0.134 & ,000c \\
\hline & Hacerse ilusiones & 58.40 & 9.227 & 0.122 &, $000 c$ \\
\hline \multirow{11}{*}{$\begin{array}{l}\text { Estrategias de } \\
\text { afrontamiento }\end{array}$} & Estrategia de falta de afrontamiento & 61.29 & 8.314 & 0.147 &, $000 c$ \\
\hline & Reducción de la tensión & 66.66 & 11.729 & 0.093 &, $004 c$ \\
\hline & Acción social & 55.54 & 8.768 & 0.129 &, $000 c$ \\
\hline & Ignorar el problema & 47.54 & 8.660 & 0.122 &, $000 c$ \\
\hline & Autoinculparse & 53.46 & 9.594 & 0.127 &, $000 c$ \\
\hline & Reservarlo para sí & 67.25 & 13.509 & 0.109 &, $000 c$ \\
\hline & Buscar apoyo espiritual & 63.14 & 10.868 & 0.121 &, $000 c$ \\
\hline & Fijarse en lo positivo & 57.61 & 13.995 & 0.102 & ,001c \\
\hline & Buscar ayuda profesional & 52.46 & 12.107 & 0.097 &, $002 c$ \\
\hline & Buscar diversiones relajantes & 47.35 & 13.889 & 0.138 &, $000 c$ \\
\hline & Distracción Física & 58.70 & 10.166 & 0.178 &, $000^{\mathrm{C}}$ \\
\hline
\end{tabular}

${ }^{*} p<0.05$ 


\section{Correlación entre las variables}

En la tabla 11 se aprecia una relación negativa altamente significativa entre ideación suicida y todas las estrategias del estilo de afrontamiento para la resolución de problemas: invertir en amigos íntimos (rho $=-0.562^{* *}, \quad p=0.000$ ), fijarse en lo positivo ( $r h o=-0.406^{* *}, p=0.000$ ), esforzarse y tener éxito (rho $\left.=-0.400^{* *}, p=0.000\right)$, concentrarse en resolver el problema (rho $=-$ $0.353^{* *}, p=0.000$ ), buscar pertenencia (rho=$0.311^{* *}, p=0.000$ ), buscar diversiones relajantes (rho $\left.=-0.257^{* *}, p=0.002\right)$ y distracción física (rho=$0.248^{* *}, p=0.003$ ). Este hallazgo señala que mientras las adolescentes tengan la capacidad de buscar soluciones a sus problemas (en este caso, el embarazo no planificado), obtendrán niveles bajos de ideación suicida (o la ausencia de esta) y viceversa. También se observa una relación negativa altamente significativa entre ideación suicida y las estrategias del estilo de afrontamiento en relación con los demás: buscar apoyo social (rho $=-0.346^{\star *}, \mathrm{p}<0.000$ ), buscar apoyo espiritual $\left(\right.$ rho $\left.=-0.301^{* *}, p<0.000\right)$ y buscar ayuda profesional $\left(r h o=0.306^{* *}, p<0.000\right)$. Esto demuestra que las adolescentes quienes buscan apoyo en otras personas (amigos, familia, pareja, personas religiosas y profesionales de la salud mental, presentarán menores niveles de ideación suicida y viceversa. Sin embargo, se evidenció que no existe una correlación entre ideación suicida y la estrategia de acción social, (rho $=0.071, p=<0.402$ ), debido a que esta estrategia está vinculada con la aceptación del problema y la búsqueda de acciones para enfrentar su situación (organizando actividades, uniéndose a otras personas en la misma situación), por ende, no se asocia a la ideación suicida.

Finalmente, se encontró una correlación negativa altamente significativa entre ideación suicida y las estrategias del estilo de afrontamiento improductivo: preocuparse (rho $=-\quad 0.327^{* *} \mathrm{p}=0.000$ ), hacerse ilusiones (rho $\left.=-0.383^{* *}, p=0.000\right)$, falta de afrontamiento (rho $=-0.304^{* *} \mathrm{p}=, 0.000$ ), autoinculparse (rho=$0.292^{* *}, p=0.034$ ) y la estrategia reservarlo para sí (rho $=-0.179^{*}, p=0.000$ ). Es decir, a mayor uso de estas estrategias, menores niveles de ideación suicida, debido a que dichas estrategias son caracterizadas por evitar la situación estresante y generar alivio. Finalmente, se evidenció que no existe correlación entre ideación suicida y las siguientes estrategias: ignorar el problema ( $r h o=-0.040, p>0.636$ ) y reducción de la tensión ( $r h o=-0.068, p>0.422$ ), debido a que, utilizando la primera estrategia, se intenta negar la situación (el embarazo) y la segunda está enfocada en buscar paliativos (tomar licor, fumar, consumir drogas, etc.) que aparentemente le hagan olvidar del problema, por lo tanto, no tiene cabida la ideación suicida. Cabe señalar que dichas estrategias, por lo general, ahondan el problema cuando se deja de utilizarlas y no conduce a una solución, a diferencia de los otros estilos mencionados. Por lo tanto, existe relación negativa, significativa o altamente significativa entre ideación suicida y quince de las dieciocho estrategias de afrontamiento frente al impacto del embarazo en adolescentes.

Tabla 5

Coeficiente de correlación entre ideación suicida y estrategias de afrontamiento

\begin{tabular}{lll} 
Estrategias de afrontamiento & \multicolumn{2}{l}{ Ideación suicida } \\
\cline { 2 - 3 } Resolución del problema & rho & 0.000 \\
Concentrarse en resolver el problema & $-0.353^{* *}$ & 0.000 \\
Esforzarse y tener éxito & $-0.400^{* *}$ & 0.000 \\
Invertir en amigos íntimos & $-0.562^{* *}$ & 0.000 \\
Buscar pertenencia & $-0.311^{* *}$ & 0.000 \\
Fijarse en lo positivo & $-0.406^{* *}$ & 0.002 \\
Buscar diversiones relajantes & $-0.257^{* *}$ & 0.003 \\
Distracción física & $-0.248^{* *}$ & \\
Relacionamiento con otros & & 0.000 \\
Buscar apoyo social & $-0.346^{* *}$ &
\end{tabular}




\begin{tabular}{lll} 
Acción social & 0.071 & 0.402 \\
Buscar apoyo espiritual & $-0.301^{* *}$ & 0.000 \\
Buscar ayuda profesional & $-0.306^{* *}$ & 0.000 \\
Afrontamiento no productivo & & 0.000 \\
Preocuparse & $-0.327^{* *}$ & 0.000 \\
Hacerse ilusiones & $-0.383^{* *}$ & 0.000 \\
Falta de afrontamiento & $-0.304^{* *}$ & 0.636 \\
lgnorar el problema & -0.004 & 0.422 \\
Reducción de la tensión & -0.068 & 0.000 \\
Reservarlo para sí & $-0.179^{*}$ & 0.034 \\
Autoinculparse & $-0.292^{* *}$ & \\
\hline
\end{tabular}

**La correlación es significativa al nivel 0.01

\section{DISCUSIÓN}

Durante la etapa de la adolescencia, los jóvenes atraviesan diversos conflictos debido a sus cambios físicos y emocionales. Es en esta fase de desarrollo cuando intentan alcanzar la madurez emocional, asumen nuevas responsabilidades y enfrentan dificultades; para esto, ellos harán uso de diversas estrategias. Algunos adolescentes tendrán recursos de afronte más sólidos, sin embargo, otro grupo no habrá conseguido buenos métodos para sobreponerse a situaciones estresantes, por lo cual tendrán que recurrir a pensamientos respecto a la muerte, los cuales pueden llevarlos a realizar actos contra la salud e integridad propia.

Sumado a esto, para algunos jóvenes que están en esta etapa, el impacto de una noticia no esperada como un embarazo precoz, el divorcio de sus padres, la muerte de un familiar, una enfermedad terminal, mudanzas, entre otros, pueden perturbarlos y fomentar inestabilidad emocional, generando en ellos pensamientos suicidas, con el objetivo de solucionar sus problemas. Partiendo de esta idea, el embarazo precoz irrumpe en la vida de las adolescentes que aún no alcanzan la madurez física, mental ni emocional, presentando diversas consecuencias como el abandono del proyecto educativo, alejamiento del círculo social, entre otros; en este contexto, se presenta la necesidad de emplearse prematuramente en trabajos que, por lo general, son mal remunerados. Por otro lado, el embarazo no planeado en una adolescente puede ser la causa de serios trastornos biológicos y psicológicos, ya que el fuerte impacto emocional podría llevar a la menor a tener ideas suicidas (Pérez \& Merino, 2008).
Rey (2009) señala que ante un embarazo precoz, la adolescente se verá en la necesidad de hacer frente a tres procesos importantes: a) problemas de identidad, dependencia y autonomía; b) aceptación de cambios físicos; y c) aceptación del rol materno. Al respecto, en un estudio realizado por Valdivia y Molina (2003) se concluye que la adolescente no se encuentra preparada para asumir su maternidad, debido a que resulta chocante y frustrante asumir diferentes roles que son parte de la etapa adulta; esto la empuja a tener pensamientos de desvalorización. Por su parte, Páramo (2011) señala que cuando las adolescentes no cuentan con recursos, factores protectores o medios necesarios para afrontar una situación pueden desencadenar en actos suicidas.

Frente a ello, Bueno (2012) resalta que el afrontamiento no es una estrategia única que se aplica en todas las circunstancias, la gente afronta de manera diferente la muerte de los seres queridos, los problemas cotidianos o los traumas y retos, y las técnicas que usan a lo largo del tiempo y en distintas circunstancias van cambiando, dependiendo de la naturaleza del estresor y de cada situación en particular. A su vez señala que el suicidio es un factor multicausal y se presenta sin distinguir raza, edad o sexo. Las personas que optan por dar solución a los problemas, de esta manera, comienzan a tener ideas suicidas por varios días. Poco después, al no tener estrategias de afrontamiento, la ideación suicida se intensifica hasta concretarse atentando contra su vida. Incluso, si esto no se logra, el riesgo inmediato de suicidio se encuentra latente y en la mayoría es alto, por lo que la persona necesita tratamiento permanente hasta bajar el riesgo. 
En los últimos años, el incremento de suicidios ha causado gran alarma, convirtiéndose este en un problema de salud pública mundial, puesto que es la tercera causa de muerte más frecuente en jóvenes de 15 a 24 años y sexta causa mortal en menores de 14 años. Para Chávez y González (2008) la ideación suicida se define como la presencia de pensamientos o fantasías acerca de la propia muerte. Otros autores la definen como todo pensamiento acerca de la voluntad de quitarse la vida, donde el sujeto menciona ideas de no querer seguir viviendo; estas expresiones pueden o no implicar una planificación del acto suicida (cómo, cuándo y dónde llevar a cabo la conducta de autodestrucción) (Robledo, 2007).

A continuación, se presenta la discusión de los principales hallazgos encontrados en la presente investigación, cuyo propósito es conocer la relación entre las estrategias de afrontamiento y la ideación suicida en gestantes adolescentes de un hospital nacional de Lima Metropolitana.

Respecto a la relación entre los estilos de afrontamiento e ideación suicida, se encontró lo siguiente:

En primer lugar, se evidencia una relación negativa altamente significativa entre ideación suicida y las estrategias del estilo de afrontamiento para la resolución de problemas: invertir en amigos íntimos (rho $=-0.562^{* *}$, $p=0.000$ ), fijarse en lo positivo (rho $=-0.406^{* *}$, $\mathrm{p}=0.000$ ), esforzarse y tener éxito (rho=$\left.0.400^{* *}, p=0.000\right)$, concentrarse en resolver el problema (rho $=-0.353^{* *}, \mathrm{p}=0.000$ ), buscar pertenencia ( $r h o=-0.311^{* *}, p=0.000$ ), buscar diversiones relajantes (rho $=-0.257^{* *}, p=0.002$ ) actividades de ocio, leer, ir al cine y distraerse físicamente (rho $\left.=-0.248^{* *}, \quad p=0.003\right)$. Este hallazgo señala que mientras las adolescentes tengan la capacidad de buscar soluciones ante sus problemas (en este caso, el embarazo no planificado), obtendrán niveles bajos de ideación suicida o la ausencia de esta. Los resultados de este estudio se asemejan a los de Reyes, Reséndiz, Alcázar y Reidl (2017) quienes señalan que las adolescentes que presentan estilos de afrontamiento dirigidos a resolver sus problemas suelen ser más eficientes, su comportamiento y estado de ánimo suele ser estable y equilibrado y son más activas para buscar soluciones. Mientras que, si las adolescentes presentan bajos niveles de estrategias en resolución de problemas, presentarán mayores niveles de ideación suicida. Estos resultados, a su vez, coinciden con lo mencionado por Clemente y González (1996) y Frydenberg y Lewis (2000) quienes señalan que las adolescentes que optan por la muerte, lo hacen porque son incapaces de afrontar sus problemas o carecen de respuestas adecuadas en los momentos en cuando son vulnerables a crecientes presiones e incertidumbre. Chang (2002) afirma que la falta de habilidad para solucionar los problemas es un factor que predispone para la ideación suicida, puesto que muchas adolescentes se verán en dificultades para manejar el embarazo de manera adecuada, considerando al suicidio como una solución real frente a su problema.

En segundo lugar, también se observa una relación negativa altamente significativa entre ideación suicida y las estrategias del estilo de afrontamiento en cuanto a la relación con los demás: buscar apoyo social (rho $=-0.346^{* *}$, $\mathrm{p}<0.000$ ), buscar apoyo espiritual (rho=$\left.0.301^{* *}, p<0.000\right)$ y buscar ayuda profesional $\left(\right.$ rho $\left.=0.306^{* *}, p<0.000\right)$. Esto demuestra que las adolescentes que buscan apoyo en otras personas (amigos, familia, pareja, personas religiosas y profesionales de la salud mental) presentan menores niveles de ideación suicida. Esto coincide con lo referido por Mitchel (1969) quien afirma que la búsqueda de redes de apoyo social sirve de contención a los adolescentes para afrontar exitosamente las situaciones de la vida cotidiana. De la misma forma, Jersild (1968) postuló que para los adolescentes el área social tiene relevancia significativa, pues consideran necesaria la relación con los amigos y amigas. Por su parte, Huapaya (2009) afirma que los adolescentes, con mayor frecuencia, afrontan el estrés buscando apoyo y aceptación en los demás.

Investigaciones propuestas por Bueno (2012) también sustentan que las estrategias como Buscar apoyo social (As), Invertir en amigos íntimos (Ai) y Buscar ayuda profesional (Ap) (ante momentos de tensión el recurrir a los padres, amigos cercanos u otras personas para poder resolver los problemas o buscar comprensión) contribuye al restablecimiento del equilibrio y un nivel óptimo de satisfacción. Asimismo, la búsqueda de un profesional, resulta una estrategia activa, pues se busca información o consejo para idear la manera de resolver el problema. También Acevedo, López, y Manjarrez (2017) manifiestan que el apoyo social es de vital importancia para encarar la situación y minimizar el riesgo de suicidio en 
situaciones de crisis. En este sentido, Alejo y Espinoza (2015) señalan que las adolescentes, frente al embarazo, presentan la necesidad de la búsqueda de apoyo espiritual, apoyo social y apoyo profesional. El buscar soporte en personas e instituciones cuando se está en una situación de tensión es considerado algo positivo y adaptativo para afrontar los problemas (Reyes et al., 2017), es decir, que cuando las adolescentes busquen orientación de otros, presentarán menor riesgo de manifestar emociones, pensamientos y conductas suicidas.

Por otro lado, se evidenció que no existe una correlación entre ideación suicida y la estrategia "acción social", que tiene que ver con organizar o participar en grupos de apoyo con la misma problemática $(\mathrm{rho}=0.071, \mathrm{p}=<0.402)$. Es decir, la presencia de esta estrategia no está vinculada con la existencia de los pensamientos suicidas, ya sea por el poco uso de estos o porque la ideación suicida está influenciada por aspectos netamente personales, como desvalorización, desesperanza y no necesariamente por los aspectos sociales y el contexto.

Finalmente, se encontró una correlación negativa altamente significativa entre ideación suicida y las estrategias del estilo de afrontamiento no productivo: preocuparse (rho $=-0.327^{* *} p=0.000$ ), hacerse ilusiones (rho $=-0.383^{* *}, p=0.000$ ), falta de afrontamiento (rho $=-0.304^{* *} p=, 0.000$ ), autoinculparse (rho=$\left.0.292^{* *}, p=0.034\right)$ y la estrategia de reservarlo para sí $\left(r h o=-0.179^{*}, p=0.000\right)$. Reyes et al. (2017) manifiestan que dichas estrategias de evitación cognitiva, conductual, de pensamiento basado en ilusiones están asociadas a presentar sintomatología suicida. Por otro lado, Fantin et al. (2005) sugieren que los adolescentes con un estilo de afrontamiento improductivo, es decir, que tienden a preocuparse, autoinculparse y reservarse los problemas pueden ser frágiles emocionalmente y exhiben marcados cambios de conducta, generando desconcierto en los círculos de personas que les rodean. Otra característica que muestran es la desconfianza en sus propias capacidades, lo cual es evidencia de un sentimiento de inseguridad y desamparo.

Sin embargo, se evidenció que no existe correlación entre ideación suicida y las siguientes estrategias: ignorar el problema (rho $=-0.040$, $p>0.636$ ) y reducción de la tensión (rho $=-0.068$, $p>0.422$ ). Romero et al. (2010) postulan que no existen estrategias de afrontamiento mejores ni peores en sí mismas. Debido a su complejidad, se tendría que analizar su éxito o fracaso en función de diversos factores como el contexto, las características de la situación generadora de estrés, el momento vital del joven, los objetivos que se persiguen con determinado comportamiento y el apoyo social, entre otros. De otro lado, Bueno (2012) considera que las estrategias "ignorar el problema" y "reducción de la tensión" están consideradas como afrontamientos disfuncionales, sin embargo, logran calmar momentáneamente la tensión, aunque no favorezcan la adaptación a largo plazo; pues, debido a que niegan la existencia del problema, sienten un alivio momentáneo, pero la angustia vuelve después.

De lo presentado anteriormente, se concluye que existe relación negativa, significativa o altamente significativa entre la ideación suicida y quince de las dieciocho estrategias de afrontamiento del embarazo en adolescentes. Estas dos variables cobran importancia para la existencia de las conductas o pensamientos suicidas; con mayor frecuencia en las mujeres que pasan por determinados momentos de angustia (Serrano \& Flores, 2005). Este resultado es corroborado por el estudio de Cárdenas (2016) cuyos resultados presentan correlaciones significativas entre tres estilos de afrontamiento e ideación suicida: ideación suicida y resolver los problemas (rho $=0.133 \mathrm{p}<0.05)$, ideación suicida y referencia a otros $(r h o=0.159, p<0.05)$ e ideación suicida y afrontamiento improductivo $(r h o=0.262 \mathrm{p}<0.05)$. Estos resultados, a su vez, coinciden con los de la investigación de Bueno (2012) quien señala que, a menor uso de las estrategias de afrontamiento, el riesgo de suicidio será alto, por lo que el mayor problema que enfrentan las adolescentes, que cometen suicidio o lo intentan, es el de carecer de respuestas de afrontamiento suficientes o efectivas para superar dichas situaciones.

\section{Declaración de financiamiento y de conflictos de interés:}

El estudio fue financiado por los autores, quienes declaran no tener conflictos de interés.

\section{Correspondencia}

Gerson Hilaquita Cruz.

Dirección: Hospital Honorio Delgado Hideyo Noguchi, Lima, Perú

Correo electrónico: gehic@upeu.edu.pe 


\section{REFERENCIAS}

Beck, A., Kovacs, M., \& Weissman, A. (1979). Assessment of suicidal intent: the scale for suicide ideation. Jornual of Consulting and Clinical Psychology, 47(2), 343-352. Recuperado de: https://www.researchgate.net/ publication/22673360_Assessment_of_suicidal_ ideation_The_Scale_for_Suicide_Ideation.

Bueno, V. (2012). Estrés, estrategias de afrontamiento y riesgo suicida presente en personas que han realizado intentos autolíticos y acuden al servicio de emergencia del Hospital General. Universidad Central de Ecuador. (Tesis para la obtención de título de Psicología).Ecuador.

Recuperado de: http://www.dspace.uce.edu.ec/ bitstream/25000/1689/1/T-UCE-0007-52.pdf

Canessa, B. (2002). Adaptación psicométrica de las Escalas de afrontamiento para adolescentes de Frydenberg y Lewis en un grupo de escolares de Lima Metropolitana.

Redalyc, 2 (5), 191-233

Recuperado de: http://www.redalyc.org/articulo. oa?id=147118132008

Cassaretto, M.; Chau, C.; Oblitas, H.; \& Valdéz, N. (2003). Estrés y afrontamiento en estudiantes de psicología. Revista de Psicología de la PUCP, 21 (2) ,364-392. Recuperado de: https://dialnet. unirioja.es/descarga/articulo/994031.pdf.

Clemente, M. y González, A. (1996). Suicidio: una alternativa social. Madrid: Biblioteca Nueva.

Chang, E. (2002). Predicting suicide ideation in an adolescent population:examining the role of social problem solving as a moderator and a mediator. Personality and individual differences, 32(7), 1279-1291.

Chávez, M., \& González, I. (2008). Presencia de la depresión perinatal y la relación madre- infante. Estrategia metodológica para una mirada sociocultural. Revista Diversitas - Perspectivas en Psicología, 4(1), 1794-9998. Recuperado de: http://revistas.usantotomas.edu.co/index.php/ diversitas/article/viewFile/196/309

Della, M. (2006). Estrategias de afrontamiento (coping) en adolescentes embarazadas. Revista Iberoamericana en Educación, 38(3),1-15. Recuperado de: https://www. researchgate.net/profile/Marcelo_Della_Mora/ publication/28106772 Estr ategias de afrontamiento_coping_en_adolescentes_embarazadas escolarizadas/links/ $0912 \mathrm{f} 511 \mathrm{ad} 333 \mathrm{c} 1 \mathrm{~d} 24000000 /$ Estrategiasde-afrontamiento-coping-en-adolescentesembarazadas-escolares.

Encuesta sociodemográfica y de la Salud (ENDES, 2014). Mortalidad materna. Perú. Recuperado de: https://www.inei.gob.pe/media/ MenuRecursivo/publicaciones_digitales/Est/ Lib1211/i ndex.html.
Frydenberg, E. (1997). Adolescent coping: theoretical and research perspectives. Madrid: TEAEdiciones.

Frydenberg, E., \& Lewis, R. (2000). ACS: escalas de afrontamiento para adolescentes. Madrid: TEA.

Gómez, M. (2015). Estrategias de afrontamiento utilizadas por madres adolescentes para su adaptación. (Tesis de licenciatura). Universidad Rafael Landívar. Guatemala. Recuperado de: http://recursosbiblio.url.edu.gt/ tesiseortiz/2015/05/42/Gomez- Maria.pdf

Hernández. R., Fernández. C., \& Baptista. M., (2010). Metodología de la investigación 5ta Edición. México: McGraw.Hill.

Huapaya, B. (2009). Factores asociados a ideación suicida en adolescentes escolares de 3er, 4 to y 5to años de secundaria de instituciones educativas públicas del cono este de Lima. (Tesis para obtener la maestría). Universidad Nacional Mayor de San Marcos. Lima. Recuperado de: http://cybertesis.unmsm.edu.pe/bitstream/ cybertesis/3040/1/Huapaya_cd.pdf

Jersild, A. (1968). Psicología de la adolescencia. Madrid: Aguilar.

Lee, C., Chang, J. y Cheng, A. (2002). Acculturation and suicide: a case-control psychological autopsy study. Psychological Medicine, 32(1), 133-141. Recuperado de: https://www.researchgate. net/publication/11476778_Acculturation and suicide_A_ca se-control_psychological_autopsy_study.

Lepineux, C. (2016). Informe anual de evento de mortalidad materna. Salud de Caldas, 1(49), 1-20. Recuperado d e http://www.observatorio.saluddecaldas.gov.co/ desca/anuales/Mortalidad Materna año 2016.pdf.

Mayoral, J., Arias, R., Jalón, M., \& Gutiérrez, R. (2008). Comportamiento violento en adolescentes: su relación con las estrategias cognitivas y el rendimiento académico. Revista de Psicología Educativa, 14(1), 63-81. Recuperado de: https://dialnet.unirioja.es/servlet/ articulo?codigo $=2690860$.

Mitchel, C. (1969). Social network in urban situations. Manchester: University Press.

Navasconi, P., \& Da Silva, L. C. (2017). Conducta suicida en adolescentes y jóvenes brasileños. Revista Alternativas y Psicología, 37(1) ,823. Recuperado de: http://www.alternativas. me/attachments/article/141/01\%20\%20 $\% 20$ Conducta $\% 20$ suici da $\% 20$ en $\% 20$ adolescentes $\% 20 \mathrm{y} \% 20 \mathrm{j} \% \mathrm{C} 3 \% \mathrm{~B} 3$ venes $\% 20$ brasile\%C3\%B10s.pdf. 
Organización Mundial de la Salud (OMS, 2017). Adolescentes: riesgos para la salud $y$ soluciones. Ginebra: Recuperado dehttp:// www.who.int/mediacentre/news/releases/2017/ yearly-adolescent-deaths/es/.

Páramo, M. (2011). Factores de riesgo y factores de protección en la adolescencia: análisis de contenido a través de grupos de discusión. Terapia Psicológica, 29(1), 85-95. doi: .org/10.4067/ S0718-48082011000100009

Perú 21 (20 de enero, 2018). Mil niñas de 12 y 13 años se convierten en madres cada año en el Perú. Recuperado de https://peru21.pe/lima/ mil-ninas-12-13-anos-convierten- madres-anoperu-198013.

Rey, M. (2009) Representaciones de apego en madres adolescentes de nivel socioeconómico bajo. (Tesis para la obtención del título de Licenciado en Psicología).Pontificia Universidad Católica del Perú. Perú.

Reyes, V., Reséndiz, A., Alcázar, R., \& Reidl, L. A. (2017). Las estrategias de afrontamiento que utilizan los adolescentes ante situaciones que provocan miedo. Psicogente, 20(38), 240-255.

Robledo, P. (2007).Suicidio en adolescentes: lo que el equipo de salud debe saber. Revista Perú Pediatr, 60(1), 61-64. Recuperado de: http://sisbib.unmsm.edu.pe/BVRevistas/rpp/ v60n1/pdf/a11v60n1.pdf. Recuperado de: http:// www.psicoter.es/_arts/90_A077_12.pdf
Rojas, I. (2013). Programa de naciones de prevención del suicidio: orientaciones para su implementación: Ministerio de Salud MINSAL. Recuperado de: http://web.minsal.cl/sites/default/ files/Programa_Nacional_Prevencion.pdf

Salas, C., \& Yanqui, M. (2013). Inteligencia emocional y riesgo de ideación suicida en adolescentes de instituciones públicas del distrito de cerro Colorado de Arequipa (Tesis para obtención de título de Psicología).Universidad Nacional de San Agustín. Arequipa.

Serrano, M. \& Flores, M. (2005). Relación con los padres y apoyo social en adolescentes. (Informe de investigación no publicado).Facultad de Psicología de la niversidad Autónoma de Yucatán. México.

Sorto, E., López, B., \& Velásquez, N. (2013). Análisis de la relación entre estrategias de afrontamiento y calidad de vida en adolescentes embarazadas atendidas en hospitales públicos de la zona oriental durante el año 2013. (Tesis para optar el título de Licinciado en Psicología). Universidad de El Salvador. El Salvador. Recuperado de: http://ri.ues.edu.sv/5468/1/50107899.pdf.

Valvidia, M., \& Molina, M. (2003). Factores psicológicos asociados a la maternidad adolescente en menores de 15 años. Redalyc, 12(2), 85-109. doi: org/10.5354/0719- 0581.2003.17457.

Recibido: 16/02/18

Aceptado: 09/05/18 